\title{
Clinical comparison of choledochal cysts between children and adults
}

\author{
Sung Min Jang ${ }^{1}$, Beom Seok Lee ${ }^{1}$, Kun Kuk Kim¹, Jung Nam Lee ${ }^{1}$, Yang Seo Koo², \\ Yeon Suk Kim${ }^{2}$, Hyeon Young $\mathrm{Kim}^{3}$, and Yeon Ho Park ${ }^{1}$
}

\author{
Departments of ${ }^{1}$ Surgery and ${ }^{2}$ Internal Medicine, Gachon University of Medicine and Science, Gil Hospital, \\ Incheon, ${ }^{3}$ Department of Pediatric Surgery, Seoul National University Hospital, Seoul, Korea
}

\begin{abstract}
Backgrounds/Aims: Choledochal cyst of the bile duct is characterized by cystic dilatation of the intra- or extrahepatic bile ducts. It is a relatively uncommon disease and there is still much controversy regarding its etiology as being congenital or acquired. Methods: The medical records of 60 patients who underwent surgical treatments for choledochal cyst between April 1995 and April 2009 at the Gachon University Gil Hospital were reviewed retrospectively. To compare the clinical characteristics, patients under 19 years of age were grouped into children and the others were grouped into adults. Results: Of the overall 60 patients, 24 were grouped into children and 36 were grouped into adults. Female predominance was common in both groups $(\mathrm{M}: \mathrm{F}=1: 6.5)$. The most common clinical symptom was abdominal pain $(73.3 \%)$ in both groups. Children had remarkable jaundice $(33.3 \%$ vs. $0 \%)$ and gastrointestinal symptoms including nausea and vomiting. Fever and chills were more common in children because of the associated complications of cholangitis. According to the Todani classification, type I was the most common form of choledochal cyst in both groups, and type IVa was significantly more common in children than adults $(45.3 \%$ vs. $16.7 \%)$. Thirty patients $(50 \%)$ had anomalous pancreaticobiliary ductal union which was confirmed by preoperative imaging studies or intraoperative cholangiography. All patients except for one child underwent cyst excision with hepaticojejunostomy. Conclusions: There was no significant difference in the clinical characteristics of choledochal cysts between children and adults. However, combined diseases especially bile duct malignancy were common in aged patients, early detection and more aggressive surgery is necessary for patients with choledochal cysts. (Korean J Hepatobiliary Pancreat Surg 2011;15:157-163)
\end{abstract}

Key Words: Choledochal cyst; Children; Adults; Malignancy

\section{INTRODUCTION}

Choledochal cyst is characterized by cystic dilation of the intrahepatic or extrahepatic biliary system and is known to have a high incidence rate in Asians and females. Since Vater reported the first case of this disease in 1723 , about more than $30 \%$ of cases have been reported in Japan and a relatively less number of cases have been found in Korea. It is a surgical disease with main symptoms including jaundice, abdominal pain and a mass in the right upper quadrant and is commonly found among infants and children. However, about $20-40 \%$ of the patients do not develop any symptoms until they become adults, which is the main cause for delay in its diagnosis. ${ }^{1}$ While it has been recently known that there is a high risk of a malignant tumor with an increase in age, this concept is gaining more attention in anomalous pancreaticobiliary ductal union (APBDU) which has a close relationship with other malignant tumors of the biliary system, and was found in most of the patients. ${ }^{2,3}$ A great number of adult patients visit the hospital to receive treatment for choledochal cyst after it is incidentally detected during the examination of the hepatobiliary system. Despite the fact that some of the literatures differentiate the adults and children from 10-15 years of age for convenience, the consistent standard of distinction between ages is not clear. $^{4-6}$ For this reason, there have been a relatively less number of studies which have compared the clinical features of choledochal cysts between children and adults, and the standard for diagnosis and treatment policies for

Received: May 2, 2011; Revised: August 1, 2011; Accepted: August 16, 2011

Corresponding author: Yeon Ho Park

Department of Surgery, Gachon University of Medicine and Science, Gil Hospital, 1198, Guwol-dong, Namdong-gu, Incheon 405-760, Korea Tel: +82-32-460-8418, Fax: +82-32-460-3247, E-mail: pyh@gilhospital.com 
each of these have not been established as yet. The authors aimed at providing help for future diagnosis of choledochal cyst and treatment of patients with choledochal cysts by separating and identifying the unique clinical features of choledochal cysts between children and adults who underwent surgery at the department of surgery of the Gachon University Gil Hospital.

\section{METHODS}

In this study, children and adults were divided by age into two groups; children (under 19 years of age) and adults (above 19 years of age) and the clinical features of each group were retrospectively investigated in a total 69 patients who underwent surgery at the Gachon University Gil Hospital between April 1995 and April 2009. Finally in the 60 subjects whose complete medical records were available, the parameters of age and sex at the time of diagnosis, change of symptoms according to age, pre- and post-operative laboratory findings, type of cyst, type of APBDU, accompanying disease and complications before surgery, methods of surgical treatment, postoperative complications and prognosis were compared and analyzed. Todani classification system was used to determine the anatomic type of choledochal cyst and a new classification system by Komi et al. ${ }^{7}$ was used for APBDU and the standard measurement of the length of the common duct was performed as suggested by Guelrud et al. ${ }^{8}$ Windows SPSS 15.0 ver. was used for an analysis of study results to compare the two groups using t-test, chi-square test and Fisher's exact test and statistical significance was determined based on $p$-value $<0.05$.

\section{RESULTS}

\section{Age and sex distribution}

The age distribution of the 60 patients varied widely from 27 days after birth to 71 years of age. Based on the standard age of 19 years, 24 patients were grouped into children and 36 into adults (children : adults=1 : 1.5), with adults being slightly more in number. With regard to gender, 8 were males and 52 were females. There were 4 boys and 20 girls in the children group (1:5) compared to 4 males and 32 females in the adults group (1:8).

\section{Clinical symptoms and physical examination findings}

The most common symptom was abdominal pain in $73.3 \%$ of the patients. Abdominal pain was noted in $79.1 \%$ of the children and $69.4 \%$ of the adults, showing no significant difference between the two groups ( $p=0.558)$. The manifestation of jaundice showed the most significant difference between the two groups, with $33.3 \%$ of the children having jaundice and no cases of jaundice among the adults in the initial diagnosis $(p=0.0001)$. Other symptoms which showed a notable difference between the children and adults groups were nausea and vomiting, with $54.2 \%$ of the children versus $16.7 \%$ of the adults presenting with nausea and vomiting $(p=0.004)$. While $20 \%$ of children had cough and fever due to an upper respiratory tract infection since the occurrence of upper respiratory tract infection followed by cholangitis is common in children, however no such symptoms were seen in the adults $(p=0.008)$. There was no significant difference between the children and adults groups (15\% vs. 20.8\%) with respect to the main cause of their visit to the hospital for an abdominal mass, it was one of the three major manifestation of choledochal cysts (Table 1).

\section{Period of medical history and main laboratory findings}

The period of the medical history varied from 4 days to 20 years at the maximum. Most children (90.2\%) had a less than 1-month period of medical history while adults were $66.4 \%$ The main clinical tests before surgery were determined by applying normal values of each age group based on laboratory test findings during hospitalization before surgery. Both groups showed overall changes in the laboratory values depending on the occurrence of cholangitis. The most common abnormalities were the increase in serum alkaline phosphatase as was seen in 18 children (75\%) and 22 adults (61\%). The increase in serum hepatic enzyme levels showed a significant difference between the children and adults groups respectively (12 [50\%] vs. 8 [22\%]). The increase in serum bilirubin level along with different symptoms of jaundice was seen in 22 children (91.7\%) and 2 adults (5.5\%) respectively. The difference in the level of increased leucocyte count was not significant between the children and adults (8 [33.3\%] vs. 12 [33.3\%]) (Table 2). 
Table 1. Clinical symptoms

\begin{tabular}{lrrrll}
\hline \multicolumn{1}{c}{ Symptoms } & $\begin{array}{c}\text { Overall, } \\
\mathrm{n}(\%)\end{array}$ & \multicolumn{1}{c}{$\begin{array}{c}\text { Child, } \\
\mathrm{n}(\%)\end{array}$} & $\begin{array}{l}\text { Adult, } \\
\mathrm{n}(\%)\end{array}$ & $p$-value \\
\hline Abdominal pain & $44(73.3)$ & $19(79.1)$ & $25(69.4)$ & 0.558 \\
Jaundice & $8(13.3)$ & $8(33.3)$ & $0(0)$ & 0.0001 \\
Fever, chills & $17(28.3)$ & $10(41.7)$ & $7(19.4)$ & 0.086 \\
Nausea, vomiting & $19(31.7)$ & $13(54.2)$ & $6(16.7)$ & 0.004 \\
Cough, fever & $5(8.3)$ & $5(20.8)$ & $0(0)$ & 0.008 \\
Abdominal mass & $9(15)$ & $5(20.8)$ & $4(11.1)$ & 0.468 \\
\hline
\end{tabular}

Table 2. Abnormal laboratory findings

\begin{tabular}{lccc}
\hline Laboratory findings & Children (\%) & Adults (\%) & Total (\%) \\
\hline Increased ALP & $18(75)$ & $22(61)$ & $40(66.7)$ \\
Increased serum & $12(50)$ & $8(22)$ & $20(33.3)$ \\
$\quad$ AST/ALT & $22(91.7)$ & $2(5.5)$ & $24(40)$ \\
$\begin{array}{l}\text { Increased total } \\
\text { bilirubin }\end{array}$ & $5(20.8)$ & $6(16.7)$ & $11(18.3)$ \\
Increased amylase & $8(33.3)$ & $12(33.3)$ & $20(33.3)$ \\
Leukocytosis &
\end{tabular}

ALP, alkaline phosphatase; AST, apartate aminotransferase; ALT, alanine aminotransferase

\section{Methods for diagnosis}

The most frequently used method for diagnosis before surgery was ultrasonography and computed tomography (CT) in $79.2 \%$ and $66.7 \%$ of the children $(p=0.085)$ and $58.3 \%$ and $66.7 \%$ of the adults $(p=1.000)$ respectively, without any significant difference between the two groups. A small number of children (4.2\%) and most of the adults $(77.8 \%)$ received endoscopic retrograde cholangiopancreatography (ERCP) $(p=0.000)$. While hepatobiliary scan was performed in $8.3 \%$ of the children, it was not at all performed in the adults $(p=0.054)$ (Table 3).

\section{Anatomic types of choledochal cysts}

The most common type of choledochal cysts according to the Todani classification was Type Ia $(36.7 \%)$ as seen in $25.0 \%$ and $44.4 \%$ of the children and adults respectively (Table 4). Type Ic and Type IVa were observed in $28.3 \%$ of the total patients, with $25.0 \%$ and $45.3 \%$ in the children and $30.6 \%$ and $16.7 \%$ in the adults respectively. Type Ib, Type II, and Type III were each seen in one adult and Type IVb was seen in one child. Type V (Calori disease) was not seen in both the children
Table 3. Preoperative diagnostic procedures

\begin{tabular}{lcccll}
\hline \multicolumn{1}{c}{ Symptoms } & $\begin{array}{c}\text { Overall, } \\
\mathrm{n}(\%)\end{array}$ & $\begin{array}{c}\text { Child, } \\
\mathrm{n}(\%)\end{array}$ & $\begin{array}{c}\text { Adult, } \\
\mathrm{n}(\%)\end{array}$ & $p$-value \\
\hline Ultrasonography & $40(66.7)$ & $19(79.2)$ & $21(58.3)$ & 0.085 \\
CT & $40(66.7)$ & $16(66.7)$ & $24(66.7)$ & 1.000 \\
MRI & $13(21.7)$ & $4(16.7)$ & $9(25)$ & 0.749 \\
ERCP & $29(48.3)$ & $1(4.2)$ & $28(77.8)$ & 0.0001 \\
MRCP & $11(18.3)$ & $2(8.3)$ & $9(25)$ & 0.174 \\
Hepatobiliary scan & $3(5)$ & $3(12.5)$ & $0(0)$ & 0.054 \\
PTC & $6(10)$ & $4(16.7)$ & $2(5.6)$ & 0.196 \\
\hline
\end{tabular}

CT, computed tomography; MRI, magnetic resonance imaging; ERCP, endoscopic retrograde cholangiopancreatography; MRCP, magnetic resonance cholangiopancreatography; PTC, percutaneous transhepatic cholangiography

Table 4. Types of choledochal cysts according to the Todani classification

\begin{tabular}{lccc}
\hline Cyst type & Overall, n (\%) & Child, n (\%) & Adult, n (\%) \\
\hline Type Ia & $22(36.7)$ & $6(25.0)$ & $16(44.4)$ \\
Type Ib & $1(1.7)$ & $0(0)$ & $1(2.8)$ \\
Type Ic & $17(28.3)$ & $6(25.0)$ & $11(30.6)$ \\
Type II & $1(1.7)$ & $0(0)$ & $1(2.8)$ \\
Type III & $1(1.7)$ & $0(0)$ & $1(2.8)$ \\
Type IVa & $17(28.3)$ & $11(45.3)$ & $6(16.7)$ \\
Type IVb & $1(1.7)$ & $1(4.2)$ & $0(0)$ \\
Type V & $0(0)$ & $0(0)$ & $0(0)$ \\
\hline
\end{tabular}

and adults.

\section{Anomalous pancreaticobiliary ductal union (APBDU) and common channel}

The incidence of APBDU based on the results of cholangiography done during preoperative $\mathrm{CT}$, MRCP, ERCP and percutaneous transhepatic cholangiography (PTC) and during surgery was $50 \%$ (30 of 60 ). APBDU was identified in only half of the patients; in 12 children and 18 adults, and the diagnosis of APBDU was unclear in 18 cases $(30 \%)$. The remaining 12 patients were diagnosed as having common channel without APBDU on pre- and post-operative tests.

The length of the common channel was studied in the total patients who underwent ERCP, MRCP and surgical cholangiography and was measured in 27 patients. The average length of the common channel was $1.76 \mathrm{~cm}$ and the length varied between $0.7-3.8 \mathrm{~cm}$.

\section{Surgical treatments}

Complete choledochal cyst resection and Roux-en-Y 
hepaticojejunostomy were performed in $23(95.8 \%)$ of the 24 children and cystjejunostomy was performed in the remaining 1 patient. All 36 adults underwent choledochal cyst resection and Roux-en-Y hepaticojejunostomy without exception.

\section{Postoperative complications}

A total of 24 patients (40\%) (5 children and 19 adults) developed early complications within 30 days after surgery (Table 5). The most common complication was bleeding as was seen in 2 children (8\%) and 5 adults (13.9\%). Three adult patients with bleeding complications required reoperation while the remaining patients recovered with conservative treatment. Surgical site infection occurred in 1 child and 3 adults, and all 4 cases of severe cholangitis after surgery were seen in the adults. Other complications included biliary leakage $(n=3)$, intra-abdominal abscess $(n=3)$, and postoperative intestinal obstruction $(n=1)$. The occurrence of postsurgical death was

Table 5. Early postoperative complications

\begin{tabular}{lcll}
\hline Postoperative complications & $\begin{array}{c}\text { Total, } \\
\mathrm{n}(\%)\end{array}$ & $\begin{array}{l}\text { Child, } \\
\mathrm{n}(\%)\end{array}$ & $\begin{array}{l}\text { Adult, } \\
\mathrm{n}(\%)\end{array}$ \\
\hline Overall complications & $24(40.0)$ & $5(20.8)$ & $19(52.8)$ \\
Bleeding & $7(11.7)$ & $2(8.0)$ & $5(13.9)$ \\
Wound infection & $4(6.7)$ & $1(4.2)$ & $3(8.3)$ \\
Cholangitis & $4(6.7)$ & $0(0.0)$ & $4(11.1)$ \\
Bile leakage & $3(5.0)$ & $1(4.2)$ & $2(5.6)$ \\
Intraabdominal abscess & $3(5.0)$ & $0(0.0)$ & $3(8.3)$ \\
Adhesion & $1(1.7)$ & $1(4.2)$ & $0(0.0)$ \\
Septic shock & $1(1.7)$ & $1(4.2)$ & $0(0.0)$ \\
Seroma & $1(1.7)$ & $0(0.0)$ & $1(2.8)$ \\
Pancreatitis & $1(1.7)$ & $0(0.0)$ & $1(2.8)$ \\
Transverse colon & $1(1.7)$ & $0(0.0)$ & $1(2.8)$ \\
perforation & $1(1.7)$ & $0(0.0)$ & $1(2.8)$ \\
Peri-wound petechiae & & &
\end{tabular}

identified in 1 child and 1 adult respectively. The main cause of death was postsurgical septicemia in the child, and acute renal failure due to postsurgical intra-abdominal bleeding in the adult.

The occurrence of complications within 30 days after surgery was identified in 10 patients ( 5 children and 5 adults). One child and adult each developed surgical site abscess. While intraheptic biliary stricture and melena, pneumobilia, and intraheptic bile duct stones were found in one adult each, keloid scar around the wound, repeated cholangitis due to biliary infection, postsurgical dysuria, and surgical site abscess were identified in one child each.

\section{Follow-up and patients with biliary malignant tumor}

A total of 4 patients (11\%) showed the development of choledochal cyst-related biliary tract cancer. A malignant tumor was seen in three patients on the histopathologic examination performed after the choledochal cyst resection (Table 6). They all were female adults, with two female adults having Type Ia and one having Type IVa form according to the Todani classification. The diagnosis was adenocarcinoma without lymph node metastasis in all three cases. Among these three female adults, one patient had postsurgical positive resection margin around the proximal biliary duct (Table 6). The patient who had a positive resection margin died after conservative treatment due to a progressive tumor found during the follow-up at around 4 months after surgery.

One patient developed a new malignant tumor during the postoperative follow-up period. The patient complained of abdominal pain, abdominal mass, and fever and chills during the initial operation and was diagnosed as Type IVa form of choledochal cyst according to the

Table 6. Characteristics of the three patients with choledochal cyst-related malignant tumor at the time of surgery

\begin{tabular}{|c|c|c|c|}
\hline $\begin{array}{l}\text { Patient } \\
\text { sex and age (years) }\end{array}$ & $\mathrm{F} / 38$ & $\mathrm{~F} / 53$ & $\mathrm{~F} / 54$ \\
\hline Symptoms & No symptoms & No symptoms & Abdominal pain, fever \\
\hline Operative intervention & $\begin{array}{l}\text { Cyst excision and } \\
\text { hepaticojejunostomy }\end{array}$ & $\begin{array}{l}\text { Cyst excision and } \\
\text { hepaticojejunostomy }\end{array}$ & $\begin{array}{l}\text { Cyst excision and } \\
\text { hepaticojejunostomy }\end{array}$ \\
\hline Type of cyst & Ia & Ia & $\mathrm{IVa}$ \\
\hline Pathology & Adenocarcinoma & Adenocarcinoma & Adenocarcinoma \\
\hline Size $(\mathrm{cm})$ & 2 & 6 & 2 \\
\hline Lymph node metastasis & No & No & No \\
\hline $\begin{array}{l}\text { Tumor involvement of the } \\
\text { resection margin }\end{array}$ & Negative & Positive & Negative \\
\hline
\end{tabular}


Todani classification. Cyst resection and hepaticojejunostomy were performed at that time and no malignancy was observed on the histological examination. Five years after surgery, a diagnosis of cholangiocarcinoma around the intrahepatic bile duct in the upper area of the hepaticojejunostomy site was made. The patient died 6 months after receiving palliative chemotherapy since no surgical procedure was available according to the $\mathrm{CT}$ and bone scan results.

\section{DISCUSSION}

Choledochal cyst is a very rare disease that occurs in only 1 out of 2 million people in the western countries while it has a higher incidence rate in the eastern countries, especially a more than 10 times incidence rate in Japan, indicating an area-specific frequency. ${ }^{7,8}$ The incidence rate in Korea is on the increase due to the recent development in the diagnostic technology and a rapid growth in the subclinical adult patients through health examinations compared to 200 cases reported till the 1990s. ${ }^{9}$ Choledochal cyst is characterized by its remarkably higher incidence in the female patients together with the regional preference. In this study also, we found more cases of female patients, $83.3 \%$ of girls and $88.9 \%$ of female adults. Though the distribution of occurrence according to the age varies to a great extent, it is reported that most of the patients are infants or children below 10 years of age and only about $20-40 \%$ of cases are found in adults. ${ }^{1,8,10}$ However, this study comprised of more number of cases of adults over 19 years of age, mainly due to the institutional bias. An increased number of adult cases have been found according to the recent 5-year results. Additionally, 45 patients $(75 \%)$ have been diagnosed with this disease since 2000, which demonstrates the development of diagnostic methods accompanied by an increased incidence rate.

The main symptoms of choledochal cyst include abdominal pain, jaundice, and an abdominal mass with less than one-third of the simultaneous incidence rates of all three symptoms. Intermittent jaundice is mostly noticed in babies or infants, whereas abdominal pain is common in children and adults. ${ }^{4-6}$ Because unusual symptoms are detected with an increase in age, and it is also hard to distinguish this disease from the other biliary or pancreatic diseases, the diagnosis is either delayed or the condition is misdiagnosed on many occasions. ${ }^{5,11}$ Thus, it seems necessary to consider the diagnosis of choledochal cyst during diagnostic medical imaging for repeated pancreatitis with intermittent abdominal pain in adult patients as well as children. Although rare in children, complications of biliary stasis such as cholangitis, choledochal cyst rupture, secondary biliary cirrhosis, and portal hypertension can develop. ${ }^{6,12}$ In our experience, a pediatric patient died from septicemia and this patient had a history of cholangitis prior to surgery. Based on this, it can be interpreted that the treatment for infection, before and after surgery is critical for the patient's prognosis.

Choledochal cyst can be easily distinguished by using abdominal ultrasonography and CT scan from the other intraabdominal diseases. Particularly, it is known that Type I and Type IV can be diagnosed easily with ultrasonography only. However, it is also required to perform some diagnostic procedures such as ERCP, PTC, and hepatobiliary scan for surgery, clear identification of the type, and an anomalous pancreaticobiliary duct. Among them, ERCP not only provides a clearer understanding of the anatomic connection of the pancreaticobiliary duct than ultrasonography or CT scan, but also helps to determine the cause of the disease. ${ }^{13,14}$

However, since it is difficult to perform invasive ERCP in pediatric patients, in this study ERCP was performed in only one case. Therefore, ERCP is being increasingly replaced by a more non-invasive MRCP due to the possibility of more cases in children in the future. It is likely that MRCP will offer more imagery information although it is not comparatively commonly used in Type 1 or Type IVa forms of choledochal cysts.

The most commonly used classification of the anatomic types of choledochal cyst is the Todani classification reported in 1977. According to the Todani classification, Type I with extrahepatic biliary segmental or overall expansion is the most common and Type IV has been reportedly found more in adults than children. ${ }^{15} \mathrm{We}$ observed that Type I was found most frequently in the overall children patients $(50 \%)$ and the incidence of Type IVa was especially as high as $45.3 \%$, while Type IVb had a frequency rate of $4.2 \%$. In adult patients, the incidence of Type IV was relatively lower $(16.7 \%)$ and the incidence of Type I was higher (80.6\%), and a significantly 
more number of Type I cases were seen. However, among the Type IV cases, there were no patients who had intrahepatic biliary expansion during the postoperative follow-up. Since a majority of studies do not provide much information on incidence of the changes in the intrahepatic biliary duct after operation for the Type IVa form, taking this into consideration future studies should focus on supporting treatment standards. Based on the pre-surgical imaging studies, we divided the choledochal cysts into Type IVa if it was accompanied by intrahepatic biliary expansion. More accumulated studies in the future will be able to provide a proper diagnostic and treatment standard for follow-up with the presence of remnant intrahepatic biliary expansion in type IVa choledochal cyst, compared to the current regular post-surgical tests using abdominal CT scan.

Although simple drainage procedure was used in the past for adult choledochal cysts, total resection has been regarded as the basic treatment method since 1980s. ${ }^{16}$ There is no doubt that the cyst should be removed immediately after diagnosis to prevent recurrence of cholangitis, pancreatitis and biliary cancer. The presence or absence of accompanying malignant diseases determines the range of resection, but basically it is the type and location of choledochal cyst which determines the extent of resection, reconstruction method, and whether or not hepatic resection is to be performed. Thus, the type of choledochal cyst becomes the most critical variable. ${ }^{12,15,17}$ We carried out total resection of choledochal cyst and Roux-en-Y hepaticojejunostomy for all cases except for one child who developed septicemia with severe cholangitis.

The development of malignant tumor, which is the biggest problem in treatment of patients with choledochal cysts, tends to increase as the age increases. ${ }^{18}$ In this study also, we found three adult cases $(8.3 \%)$ which were accompanied by bile duct cancer on biopsy after the initial surgery, whereas no case of malignant tumor was found in the children. The patient's ages were 38, 53 and 54 respectively, which were notably lower than the age of patients with bile duct cancer without choledochal cyst. $^{18,19}$ One patient who developed bile duct cancer during the follow-up after an initial surgery was a 22-yearold female who had an occurrence of intrahepatic bile duct cancer in the upper area of the hepaticojejunostomy site at 5 years after surgery. She received palliative che- motherapy but died after 6 months. As seen in this case, it is necessary to pay special attention to the accompanying diseases such as bile duct cancer or intrahepatic cholangiocarcinoma, which imply a critically negative prognosis in the $20 \mathrm{~s}$ or 30 s age groups. However, further studies are required to assess the occurrence of malignant tumor in patients who underwent total resection. ${ }^{18,20}$

In conclusion, we compared the clinical manifestations of adults and children among the 60 patients with choledochal cysts who were treated for over 14 years. Although there was no significant difference in the main clinical symptoms between the two groups, jaundice was observed in a higher number of children while almost no case of jaundice was found in the adults. Four (11\%) adult patients had biliary tract cancer and two died due to the development of cancer, which indicates that choledochal cyst has a relatively close relationship to with malignant transformation, especially in aged patients.

\section{REFERENCES}

1. Gertler JP, Cahow CE. Choledochal cyst in the adult. J Clin Gastroenterol 1988;10:315-319.

2. Babbitt DP, Starshak RJ, Clemett AR. Choledochal cyst: a concept of etiology. Am J Roentgenol Radium Ther Nucl Med 1973;119:57-62.

3. Nagata E, Sakai K, Kinoshita H, Hirohashi K. Choledochal cyst: complications of anomalous connection between the choledochus and pancreatic duct and carcinoma of the biliary tract. World $\mathrm{J}$ Surg 1986;10:102-110.

4. Suh SJ, Choi SO, Park WH. Clinical analysis of choledochal cyst in childhood. J Korean Surg Soc 2003;65:564-571.

5. Moon HJ, Choi DI, Heo JS, Joh JW, Kim YI, Choi SH. Asymptomatic adult choledochal cyst. J Korean Surg Soc 2004; 66:226-230.

6. Nam YH, Jung JH, Song YT. Clinical analysis of choledochal cysts in children. J Korean Surg Soc 2008;75:328-335.

7. Komi N, Takehara H, Kunitomo K, Miyoshi Y, Yagi T. Does the type of anomalous arrangement of pancreaticobiliary ducts influence the surgery and prognosis of choledochal cyst? J Pediatr Surg 1992;27:728-731.

8. Guelrud M, Morera C, Rodriquez M, Prados J, Jaen D. Normal and anomalous pancreaticobiliary union in children and adolescents. Gastrointestinal Endoscopy 1999;50:189-193.

9. Lee JS, Lim TS, Kim YS. A clinical analysis of choledochal cyst. J Korea Surg Soc 1990;38:459-466.

10. O'Neill JA Jr, Templeton JM Jr, Schnaufer L, Bishop HC, Ziegler MM, Ross AJ 3rd. Recent experience with choledochal cyst. Ann Surg 1987;205:533-540.

11. Lee YC, Kim JS, Kim JJ, et al. Characteristics of adult-onset choledochal cyst. J Korean Surg Soc 2001;61:81-85.

12. Robertson JF, Raine PA. Choledochal cyst: a 33-year review. $\mathrm{Br}$ J Surg 1988;75:799-801.

13. Mori K, Nagakawa T, Ohta T, et al. Pancreatitis and anomalous union of the pancreaticobiliary ductal system in childhood. $\mathrm{J}$ 
Pediatr Surg 1993;28:67-71.

14. Kim MJ, Han SJ, Yoon CS, et al. Using MR cholangiopancreatography to reveal anomalous pancreaticobiliary ductal union in infants and children with choledochal cysts. AJR Am J Roentgenol 2002;179:209-214.

15. Nagorney DM, McIlrath DC, Adson MA. Choledochal cysts in adults: clinical management. Surgery 1984;96:656-663.

16. Ono J, Sakoda K, Akita H. Surgical aspect ot cystic dilatation of the bile duct. An anomalous junction of the pancreaticobiliary tract in adults. Ann Surg 1982;195:203-208.

17. Takiff H, Stone M, Fonkalsrud EW. Choledochal cysts: results of primary surgery and need for reoperation in young patients.
Am J Surg 1985;150:141-146.

18. Ishibashi $\mathrm{T}$, Kasahara $\mathrm{K}$, Yasuda $\mathrm{Y}$, Nagai $\mathrm{H}$, Makino $\mathrm{S}$, Kanazawa K. Malignant change in the biliary tract after excision of choledochal cyst. Br J Surg 1997;84:1687-1691.

19. Todani T, Tabuchi K, Watanabe Y, Kobayashi T. Carcinoma arising in the wall of congenital bile duct cysts. Cancer 1979; 44:1134-1141

20. Fujisaki S, Akiyama T, Miyake H, et al. A case of carcinoma associated with the remained intrapancreatic biliary tract 17 years after the primary excision of a choledochal cyst. Hepatogastroenterology 1999;46:1655-1659. 